\title{
PROPERTIES OF GALAXIES IN AND AROUND VOIDS
}

\author{
ULRICH HOPP \\ Universitätssternwarte München \\ Scheiner Str. 1, D 81679 München \\ email: hopp@usm.uni-muenchen.de
}

\begin{abstract}
Two surveys for intrinsically faint galaxies towards nearby voids have been conducted at the MPI für Astronomie, Heidelberg. One selected targets from a new diameter limited $\left(\Phi \geq 5^{\prime \prime}\right)$ catalog with morphological criteria while the other used digitized objective prism Schmidt plates to select mainly HII dwarf galaxies. For some 450 galaxies, redshifts and other optical data were obtained. We studied the spatial distribution of the sample objects, their luminosity function, and their intrinsic properties.

Most of the galaxies belong to already well known sheets and filaments. But we found about a dozen highly isolated galaxies in each sample (nearest neighborhood distance $\geq 3 h_{75}^{-1} \mathrm{Mpc}$ ). These tend to populate additional structures and are not distributed homogeneously throughout the voids. As our results on 'void galaxies' still suffer from small sample statistics, I also tried to combine similar existing surveys of nearby voids to get further hints on the larger structure and on the luminosity function of the isolated galaxies. No differences in the luminosity function of sheet and void galaxies could be found.

The optical and infrared properties of both samples are in the normal range for samples dominated by late-type dwarfs. Follow-up HI studies show that the isolated dwarfs in both samples have unusual high amount of neutral gas for a given luminosity.
\end{abstract}

\section{Introduction}

One of the surprising results of the first redshift surveys was the detection of empty regions in space, the voids. Some theories predicted that a homogeneously distributed population of dwarf galaxies may exist inside these voids (Dekel \& Silk, 1986), others predicts at least filaments of matter crossing the empty regions of the nearby universe (see Colberg et al, these proceedings). In 1990, we started a survey for intrinsically small and faint galaxies towards nearby voids with the idea that some selection effects introduced by the catalogs and observers might have prevented the detection of a void population (Hopp \& Kuhn, 1995). Obvi- 
ously, dwarf galaxies of all types with their faint absolute magnitudes, often rather low surface brightness (SB), and small or even vanishing apparent diameters on Schmidt survey plates are easily missed in all kind of surveys. Indeed, it is known that dwarfs dominate the universe in number $\left(\geq 65 \%^{1}\right)$, but form only a minor contribution $(\leq 10 \%)$ to the catalogs ('Zwicky', UGC) on which the early redshift surveys like the CfA (Huchra et al, 1990) and its southern counterpart (da Costa et al., 1994) were based.

Early attempts to clarify whether or not dwarfs are distributed like the giants in the standard catalogs failed as they were limited by the standard catalogs (e.g. Thuan et al, 1987). They were not able to detect real dwarf galaxies even in the distance of the nearest voids (Binggeli et al 1990, Hopp 1994). But these surveys already showed that one has to deal with really faint objects ${ }^{2}$, and therefore has to study the nearby voids $\left(v_{R} \leq 10^{4} \mathrm{kms}^{-1}\right)$. In this sense, the famous Boötes void (Kirchner et al., 1981) which attracted otherwise very important survey work (e.g. Weistrop et al, 1995, Szomoru et al. 1996), is too distant. Indeed, most of the so-far identified $\sim 60$ galaxies in the Boötes void are not dwarfs.

Here, I will report about the two Heidelberg-void surveys. I will especially describe the properties of the very isolated galaxies which we identified in and around voids. Meanwhile, other independent surveys were done, mainly by searching emission line galaxies (ELG, see Popescu et al, 1996, for references), or galaxies of low surface brightness (LSBG, e.g. Bothun et al, 1986, Mo et al, 1994, Roennback \& Bergvall, 1996, Bothun et al, 1997, Impey et al 1996). Two surveys were dedicated to morphological selection (Eder et al, 1989, Binggeli et al 1990). I will compare to these other surveys and show that a combination of the catalogs to a common data base has great potential in studying the matter distribution in and around voids. All surveys together may serve as an ideal data base to reconstruct the mass distribution from the peculiar motion of the galaxies (following e.g. da Costa et al, 1996), especially, when the gaps between them will be filled. Throughout this paper, I use $H_{0}=75 \mathrm{kms}^{-1} \mathrm{Mpc}^{-1}$.

\section{Two Heidelberg surveys}

The shape of the luminosity function of galaxies yields magnitude limited galaxy catalogs which are dominated in number by $L^{*}$ galaxies (Dickey, 1988). Increasing the magnitude limit of a catalog thus largely increases the number of $L^{*}$ galaxies at large distances while it adds only a few to the nearer dwarfs. Therefore, the strategy of a general redshift survey like the LCRS or the SLOAN ${ }^{3}$ was neither efficient nor feasible for our purpose of finding dwarfs in the redshift distance of the nearest voids. Similar arguments hold for diameter limited catalogs (Binggeli et al, 1990). Selection affects in SB can very powerful effect the results of any survey (Disney, 1976, Bothun et al. 1997). Thus, we started dedicated surveys towards a few selected nearby voids. We tried to optimize the selection for dwarf galaxies and LSBG in and around these voids (Hopp 1994, Hopp \& Kuhn 1995). The data were reported in Hopp et al. (1995, paper 1 hereafter), and Popescu et al. 1996

\footnotetext{
${ }^{1}$ At least in the $10 \mathrm{Mpc}$ sample of Kraan-Korteweg \& Tammann (Schmidt \& Boller, 1992, Karachentsev, 1994).

${ }^{2}$ E.g.: $M_{B} \geq-16^{m}, m_{B} \geq 18^{m}, S B_{0} \geq 22^{m} / \square^{\prime \prime}, r_{s} \leq 1 k p c \sim 3^{\prime \prime}$.

${ }^{3}$ For a description of these two major redshift surveys see the contributions by the two teams in these proceedings.
} 
(paper 2) while the spatial distribution of the samples in discussed in Kuhn et al (1997, paper 3), and Popescu et al (1997, paper 4).

\subsection{VOID SELECTION}

When we started, few void catalogs were available. Therefore, we used the available cone diagrams (mostly from CfA1), and selected those voids which have a diameter of $\geq 20 M p c$, and are completely free of Zwicky and UGC galaxies. We selected four fields where $|b| \geq 40^{\circ}$. Our fields cover a total of 10 voids up to $10^{4} \mathrm{kms}^{-1}$ (see paper 1,2). Later, we learned that our naively defined voids agree quit well with more objective definitions (Kauffmann \& Fairall, 1991, Saunders et al 1991, Slezak et al 1993, Lindner et al, 1995).

\subsection{SELECTION EFFECTS}

The redshift surveys available at that time (CfA, SSRS) were based on traditional catalogs (Zwicky et al., 1961-65, Nilson, 1973, Lauberts 1982) which are limited either in apparent magnitude of $\mathrm{B} \leq 15.5^{\mathrm{m}}$ or in diameters $\Phi \geq 1.0^{\prime}$. They are most severely limited in SB due to the POSS I or ESO quick B capabilities. A discussion of the structural properties of very nearby dwarfs forces one to include much smaller diameters and also objects of lower SB (Hopp, 1994; footnote 2).

We obtained deep Calar Alto $3.5 \mathrm{~m}$ prime focus images towards the center of three of our fields and establish a diameter-limited catalog down to $\Phi \geq 5^{\prime \prime}$. In the surroundings of these deep images, I surveyed the POSS down to $\Phi \geq 21^{\prime \prime}$. Figure 1 in paper 1 shows that the resulting sample fits nicely to the diameter distribution of the UGC, going to smaller values. From this calatalogue, we selected dwarf candidates by their morphology or low SB. The follow-up redshift survey revealed a high percentage $(\sim 70 \%)$ of ELG in this sample (paper 1$)$ and a higher-thanaverage rate of ELGs among the isolated galaxies. Therefore, we decided to start a second survey for ELG's in the Hamburg Quasar Survey data base (Hagen et al, 1995) which is decribed in detail by Popescu (these proceedings, paper 2). The ELG survey is only seeing limited in $\Phi$, but galaxies with large $\Phi$ in their emission regions are hard to detect on objective prism plates. Both Heidelberg void surveys cover the range $15 \leq B \leq 20.5^{m}\left(20.0 \leq \mu_{B}\left[m / \square^{\prime \prime}\right] \leq 24.5\right)$. The magnitude completeness limit of the morphological survey is $B \sim 19.0^{m}$. The

leading selection effect for the ELG sample is the equivalent width of the emission line used for selection, in our case [OIII] 5007 . The limit is $0.8 \mathrm{~nm}$ (see Popescu, this volume, and paper 4).

\subsection{THE MORPHOLOGICAL SURVEY}

This survey covered three fields towards nearby voids. A total of $\sim 175$ redshifts were obtained together with B and R CCD images of most of the galaxies (paper 1). The $\mathrm{SB}(\mathrm{r})$ of the galaxies in a subsample was obtained by Vennik et al (1996, paper 5). Half of the galaxies have redshifts above the limits of the CfA which is the bright comparison sample in our study and are therefore useless for our goal. The average surface density of all objects is about $1 / \square^{o}$. Cone diagrams of all fields and a detailed discussion of the nearest neighbor distance $D_{N N}$ distribution are the essentials of paper 3 . 


\subsection{THE EMISSION LINE GALAXY SURVEY}

The survey was obtained in four fields, some identical to the fields of the morphological survey. A total of $\sim 250$ redshifts were obtained. For most of the galaxies, we have R or B CCD images, for some both colors, which are now under study. Most of the spectra are good enough to discuss the abundances of the objects (in prep.). The data were presented in paper 2 while the spatial distribution and the details of the selection effects are discussed in paper 4 and by Popescu in these proceedings. This survey was pretty successful in finding dwarf galaxies below $v_{r} \leq 10^{4} \mathrm{kms}^{-1}$ (see Fig. 2 of Hopp \& Kuhn 1995).

\section{Spatial distribution}

For a detailed discussion, including cone diagrams, we refer to papers 3,4 as well as to Popescu in these proceedings. We were able to detect about 25 highly isolated galaxies. We call a galaxy highly isolated when $D_{N N} \geq 3.0 h_{75}^{-1} 3 M p c$ and $c z \leq 10^{4} \mathrm{kms}^{-1}$ (Fig. 1). ${ }^{4}$ All other galaxies $(\sim 95 \%)$ follow well known sheets and filaments or are situated in the background where the sampling is too sparse for any density estimates.

Many of the highly isolated galaxies tend to populate the rims of the voids. This may simply reflect the fact that late type dwarfs have the lowest clustering power and drop off with a shallower gradient from the density peaks of the sheets.

A few are located more to the central parts of the voids. These galaxies show some tendency to align themself in very sparsely populated chains or filaments which devide bigger voids into smaller entities (paper 4). Filaments which are populated only by low luminosity galaxies are an observational argument in favour of hierarchical structure formation (Vogeley et al 1996). Unfortunately, we have only very few cases at hand in our surveys, but similar conclusion were based on other ELG surveys (Lindner et et 1996). We estimated the number density contrast between the sheet galaxies and those few in the voids to be $\Delta \rho / \rho \leq 0.1$ (paper 3, $4)$.

\subsection{SIMILAR SURVEYS}

Having so few isolated galaxies in our samples, I tried to combine our surveys with other surveys which are similar in selection functions, covered field, volume, velocity and magnitude range. These are the SBS survey (Pustil'nik et al 1995), the UM- and Case surveys by Salzer (1989) and Rosenberg et al (1994), respectively. For a comparison of these different surveys see paper 2 . I call the merged dwarf sample MDS. Naturally, this merging of catalogs yields an inhomogeneous sample (Hopp, 1997). But this exercise was mainly done to convince myself that a survey which will cover the whole nothern galactic cap and link all the above mentioned surveys, including the two Heidelberg void surveys, will be the right way to proceed. A project (under the name Hamburg-SAO survey) was already started (Ugryumov et al 1997). We obtained some further 75 redshift of ELGs which are already included in the MDS.

From the same fields as the surveys, I extracted the CfA galaxies as a comparison sample. In total, the MDS covers $4750 \square^{\circ}$ and contains 1012 (and 877 CfA)

\footnotetext{
${ }^{4}$ One should not forget that $D_{N N}$ can be measured only to the next catalogued galaxy!
} 


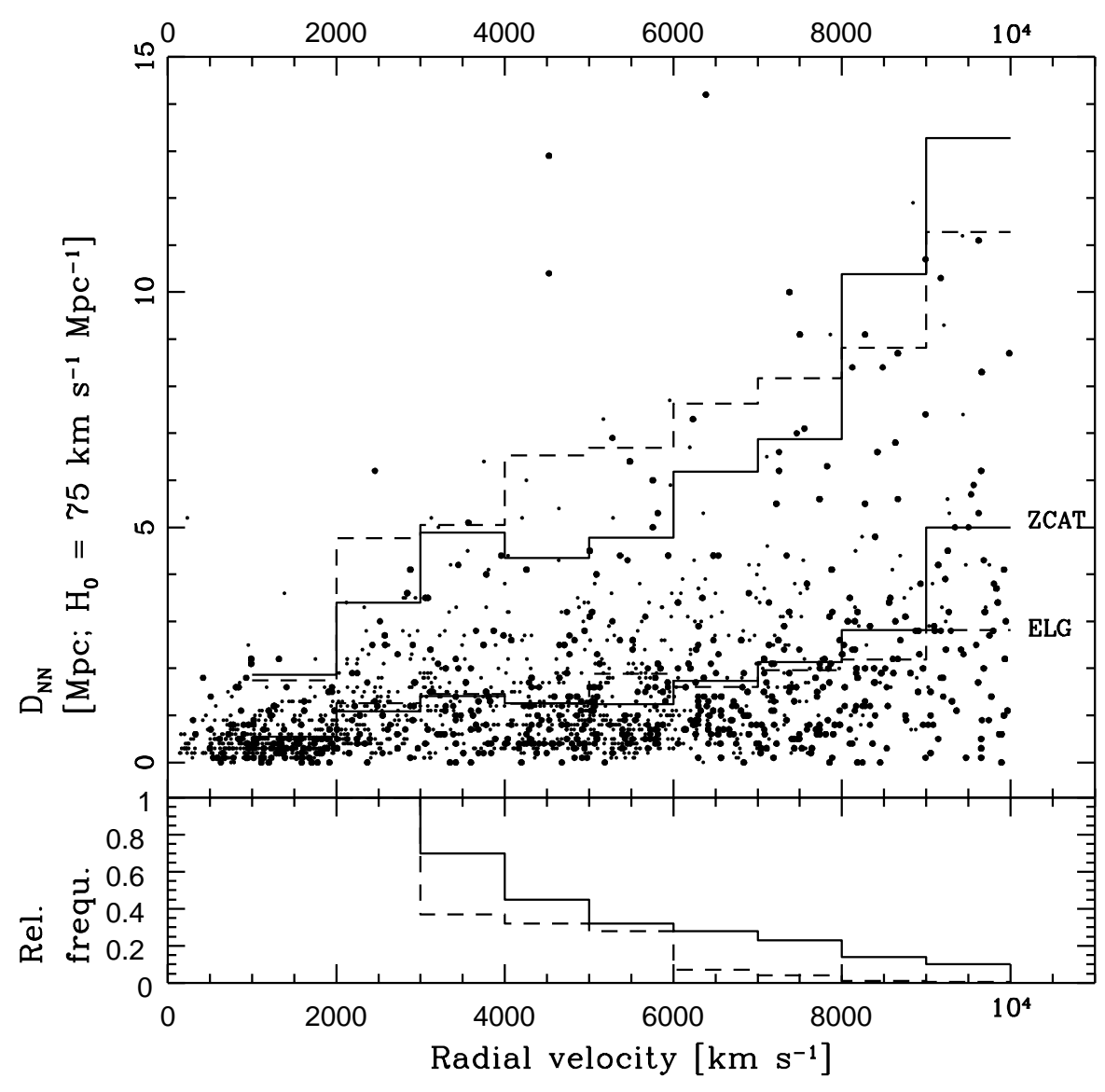

Figure 1. Top: Nearest neighborhood distance $D_{N N}$ distribution as a function of radial velocity for the MDS galaxies (big symbols) and the CfA galaxies from the same volume (small). The lower lines indicate the mean values in redshift bins of $10^{3} \mathrm{kms}^{-1}$ for the CfA (solid) and the MDS (dashed), the upper the mean values plus $3 \sigma$. Bottom: Relative frequency of galaxy numbers in MDS and CfA normalized to the bin at $2500 \mathrm{kms}^{-1}$. Obviously, the sampling decreases in both samples with increasing redshift in a similar manner. Thus, the absolute values of $D_{N N}$ at low and high redshifts can not be directly compared. Therefore, we restricted the MDS to the interval $3 . .610^{3} \mathrm{kms}^{-1}$ for comparison of high and low density galaxies with a cut between this two regimes at $3.0 h_{75}^{-1} \mathrm{Mpc}$.

galaxies within $v_{R} \leq 10^{4} \mathrm{kms}^{-1}$. All individual surveys show a few isolated galaxies as discussed by their authors.

Contrary to the authors of the ELG surveys, those who analysed the spatial distribution of LSBG (e.g. Eder et al., 1989, Mo et al, 1994, Schombert et al, 1997) found that while these LSBG are avoiding high density regions, they else still follow the structures occupied by the giants. As there is still some discrepancy in the interpretation ${ }^{5}$, and as the selection function surely differs from those of

${ }^{5}$ This comes as a little surprise as the LSBG cone diagrams shows also very isolated 
the ELG samples, I did not include these surveys into the MDS so far.

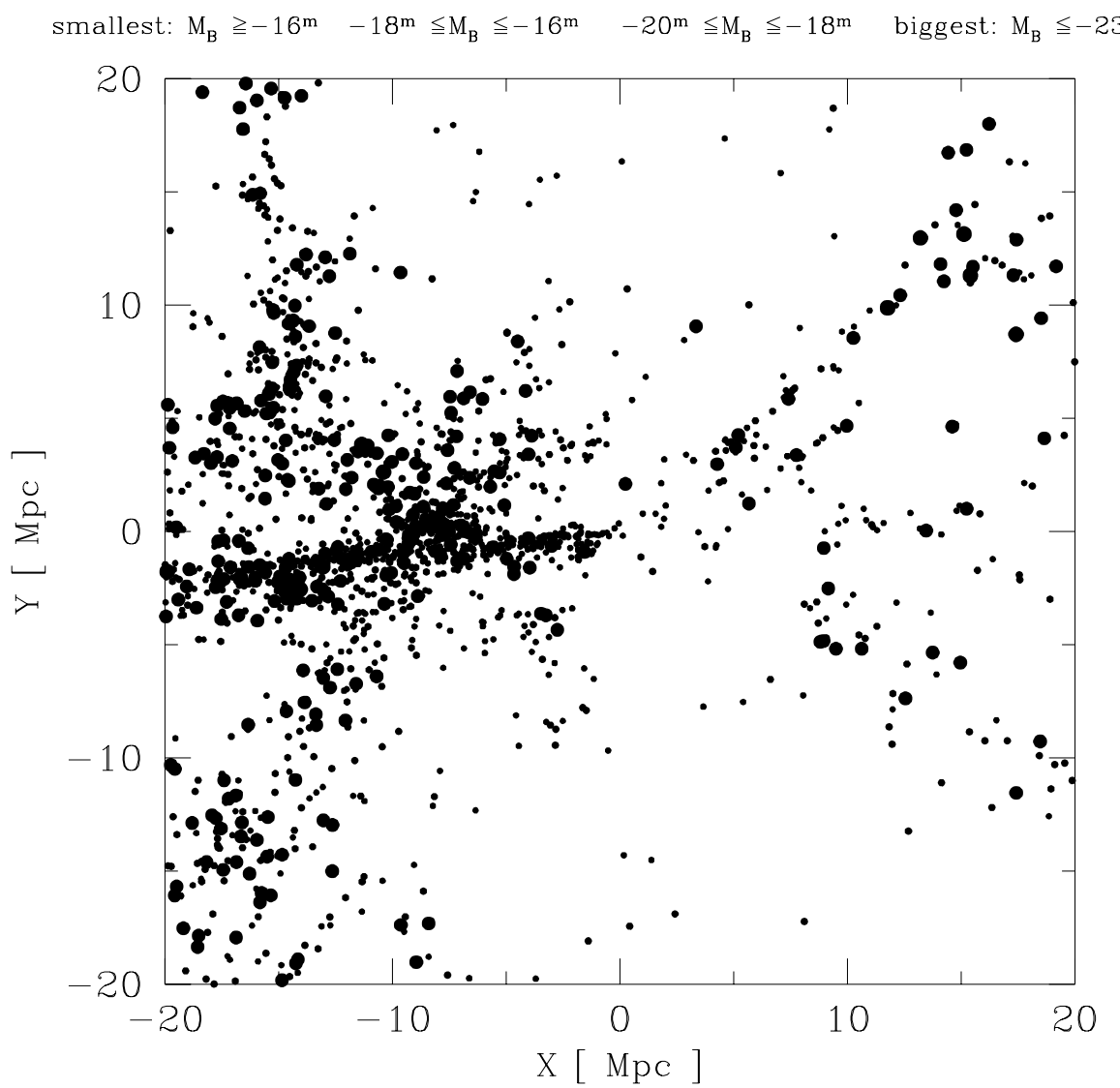

Figure 2. Spatial distribution of the merged dwarf sample and the CfA galaxies in a cube of $20 h_{75}^{-1} \mathrm{Mpc}$ side length. Third coordinate is projected. The symbol sizes indicates the absolute magnitude of the galaxies. Besides the well-known rich structures which are populated by galaxies of all luminosities, there exist regions which are only populated by low-luminosity galaxies.

Most of the MDS galaxies follow the distribution outlined by the CfA galaxies, namely the well-known structures which are defined by the massive galaxies. About 140 of the MDS galaxies are very isolated with $D_{N N} \geq 3.0 h_{75}^{-1} M p c$ (Fig. 1). These isolated galaxies are not randomly distributed, but they occupy the rims of the voids or form additional filaments which seems to be populated only by low luminosity galaxies (Fig. 2; Hopp, 1997, paper 4). Similar results have been found by Lindner et al (1996) and Vogeley et al (1994).

objects and statistical results are similar. I was always left with the impression that the discrepancy is more of semantic nature depending on the topic the authors like to address. ELG and LSBG surveys both rule out the high-biasing scenario that (irregular) dwarfs fill the voids. 


\section{Properties of isolated galaxies}

\subsection{SURFACE PHOTOMETRY}

As far as we finished the analysis, most of our objects are disk galaxies with rather blue colors, quite common for a dwarf sample. The mean scale length for the dwarfs is $2.1 \pm 0.8 h_{75}^{-1} k p c$ (paper 5). The isolated galaxies do not differ in their structural properties. In the theoretical SB versus scale length diagram shown by Dalcanton during this conference, our sheet and isolated galaxies distribute between the diameter limited UGC sample and the Virgo cluster sample in the regime of relatively small scale length and over nearly the whole range in SB (Dalcanton et al, 1997). Again, no difference is obvious between isolated and sheet galaxies. Most objects have central SB well below the 'Freeman disk'.

\subsection{LUMINOSITY FUNCTION}

I calculated the luminosity function for our sample as for the other ELG samples (Fig 3) after applying $V / V_{m}$ test completeness corrections for a volume limited sample. They all are pretty similar and show a steep faint end slope. Then, I calculated the luminosity function for the MDS within $3000 \leq c z\left[\mathrm{kms}^{-1}\right] \leq 6000$ as the completeness in this redshift range drops only slowly for dwarfs (Fig. 1). The calculation was done separately for low and high density regions (dividing line: $D_{N N}=3.0 h_{75}^{-1} \mathrm{Mpc}$ ). Within the rather poor statistics for the low density regime $(\sim 140$ galaxies $)$, the low and high density luminosity function are identical.

\subsection{HIGH HI ABUNDANCES}

We obtained HI observations for subsamples of both Heidelberg void surveys, mostly with the Effelsberg $100 \mathrm{~m}$ antenna. The detection rate was high $(\sim 67 \%$ and $\geq 50 \%$, respectively) and many galaxies show a high amount of HI gas. The line profiles indicate in most cases a dwarf galaxy. Especially, we found a trend that the HI-mass to blue luminosity ratio of dwarfs for a given luminosity increases with decreasing galaxy density, from the Virgo cluster to sheets to voids (Huchtmeier et al 1997). A first analysis of the ELG data also supports this finding. No significant deviation from the Tully-Fisher relation was found so far, including the void galaxies.

\subsection{OTHER FREQUENCIES}

With the aid of $\mathrm{NED}^{6}$ and the recently publicly available ROSAT point source catalog (Voges et al, 1996), we checked whether the galaxies found in our two surveys were detected at other frequencies, too. No galaxies from the morphological approach were detected by IRAS, they do not shine up in the ROSAT point source catalog, nor does NED list any radio continuum detection.

Contrary, about $15 \%$ of the ELG galaxies were detected by IRAS in the 60 $\mu$ band, but none of the detected sources is highly isolated. Some of them were

${ }^{6}$ The NASA/IPAC extragalactic database (NED) is operated by the Jet Propulsion Laboratory, California Institute of Technology, under contract with the National Aeronautics and Space Administration. 


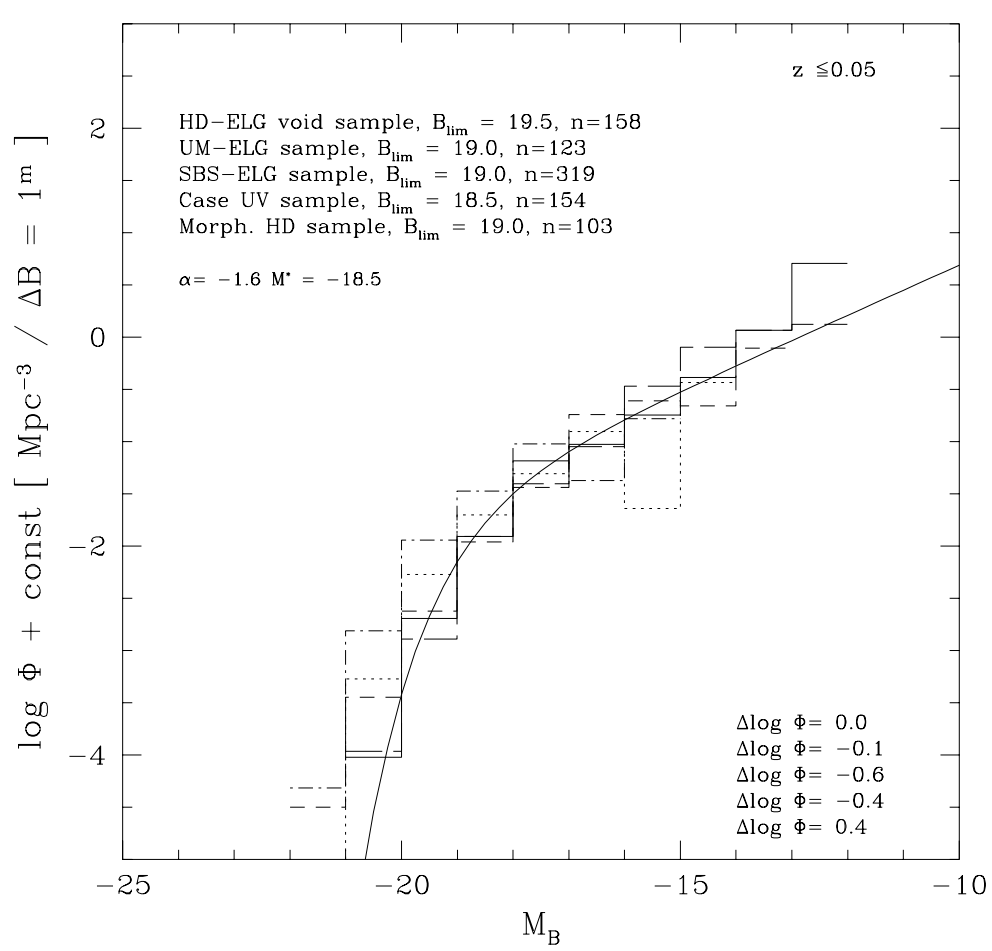

Figure 3. Luminosity function derived from our surveys and several similar sample from the literature (histogram). The line shows a Schechter function with the indicated parameters for comparison, it is not a fit. All these field dwarf samples tend to show a steep faint end slope. The differences between the samples is negligible within the given statistical and systematic errors.

also detected at $100 \mu$ or at $25 \mu$. In Fig. 4, we show a FIR-B color-color plot where the detected objects are indicated together with the general distribution for spirals and irregular dwarfs. These detections again show that our ELGs occupy mostly the dwarf parameter space. Only one single source may have been detected by ROSAT. Seven $20 \mathrm{~cm}$ FIRST detections are available from NED.

\section{Conclusions}

I presented our two Heidelberg void surveys which each identified about a dozen of highly isolated galaxies inside the voids. Some of them are distributed along the rims of the voids while others populate filaments across the voids. Some voids remain completely empty. Most of the galaxies are dwarfs with quite normal optical properties, but high HI mass. Our results agree well with the results of other surveys which detected dwarfs in and around voids. From a combination of all ELG surveys we found no evidence for a difference in the luminosity function of isolated and sheet galaxies. The number density contrast between sheets and the voids remains high $(\Delta \rho / \rho \leq 0.1)$ despite the newly identified void galaxies. 


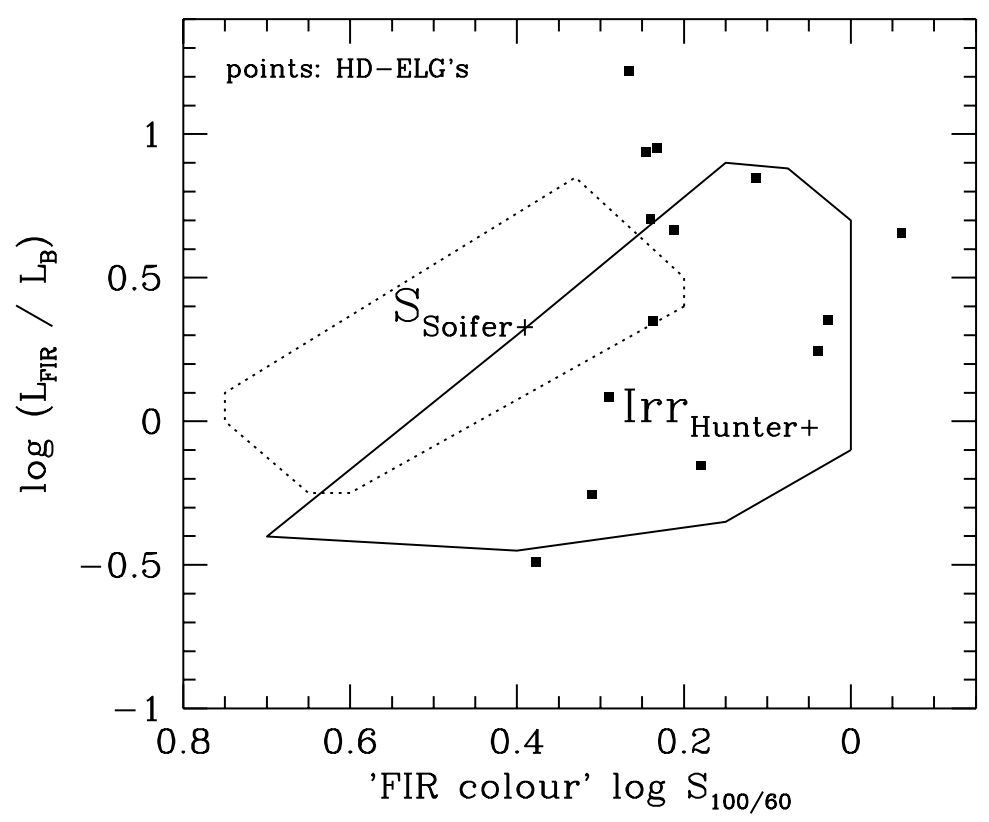

Figure 4. Plot of FIR color index in $60 \mu$ through $100 \mu$ versus ratio of FIR luminosity to B luminosity. Data from the IRAS point source catalgue for those of the Heidelberg-ELG sample which were detected by IRAS in both bands. The lines indicate the distribution regions for spiral galaxies according to Soifner et al (1987) and for irregular galaxies according to Hunter et al (1989). Most of our galaxies occupy the region of irregular dwarfs.

I like to thank for many useful discussion Drs. R. Bender, B. Binggeli, J. Einasto, H. Elsaesser, H.-J. Hagen, W.K. Huchtmeier, B. Kuhn, C.C. Popescu, S. Pulstil'nik, J. Roennback, J. Salzer, and J. Vennik. Especially, I remember with a deep and warm feeling the discussions with the late Valentin Lipovetzky who died the Sunday just before this meeting. I like to dedicate this paper to the memory of Valentin. It's a pleasure to thank Ulrich Thiele and the Calar Alto crew for the support during the many observations. The author acknowledges the support of the SFB 375 of the Deutsche Forschungsgemeinschaft.

\section{References:}

Binggeli,B., Tarenghi,M., Sandage,A., 1990, $A \xi \exists A$ 228, 42.

Bothun,C.D., Beers,T.C., Mould,J.R., Huchra,J.P., 1986, ApJ 308, 510

Bothun,C.D., Impey,C., McGaugh,S., 1997 PASP in press

da Costa, L.N., Geller,M.J., Pellegrini,P.S., Latham,D.W., Fairall,A.P., Marzke,R.O., Willmer,C.N.A., Huchra,J.P., 1994, ApJ 424, L1

da Costa, L.N., Freudling,W., Wegner,G., Giovanelli,R., Haynes,M.P., Salzer,J.J., 1996, ApJ 468, L5

Dalcanton,J., Spergel,D.N., Summers,F.J., 1997 ApJ preprint

Dekel,A., Silk,J., 1986, ApJ 303, 39

Dickey,J.M., 1988, Astron.Soc.Pac.Conf.Ser. 5, 9

Disney,M.J., 1976, Nature 263, 573 
Eder,J.A., Schombert,J.M., Deckel,A., Oemler,A., 1989, ApJ 340, 29

Hagen,H.-J., Groote,D., Engels,D., Reimers,D., 1995, A $6 \mathcal{S} A S$ 111, 195

Hopp, U.: 1994, in: G.Meylan and P.Prugniel (eds.), Dwarf Galaxies ESO Conf. Proceedings 49, 37

Hopp,U., 1997 Proc. Symp. IAU 179, in press

Hopp,U., Kuhn,B., Thiele,U., Birkle,K., Elsässer,H., Kovachev,B., (paper 1) 1995

$A E S A S \mathbf{1 0 9}, 537$

Hopp,U., Kuhn,B. 1995 Reviews in Modern Astronomy, 7, 277

Huchra,J.P., Geller,M.J., de Lapparent,V., Corwin,H.G. 1990) ApJS 72, 433

Huchtmeier,W.K., Hopp,U., Kuhn,B. 1997 A $\mathscr{H} A$ accepted

Hunter,D., Gallagher,J.S., Rice,W.L., Gillett,F.C., 1989, ApJ 336, 152

Impey,C.D., Sparberry,D., Irwin,M.J., Bothun,G.D., 1996, ApJS 105, 209

Karachentsev, I.D., 1994, A\&Ap Transactions 6, 1

Kauffmann,G., Fairall,A.P., 1991 MNRAS 248, 313

Kirchner,R.P., Oemler,A., Schechter,P.L., Schectman,S.A.: 1981, ApJ 248, L57

Kuhn,B., Hopp,U., Elsässer,H. (paper 3, 1997 A $\mathscr{\}} A$ 318, 405

Lauberts,A., 1982, The ESO/Uppsala Survey of the ESO (B) Atlas, ESO, München. Lindner,U., Einasto,M., Einasto,J., Freudling,W., Fricke,K., Lipovetsky,V., Pustilnik,S., Izotov,Y., Richter,G., $1996 A \xi \exists A$ 314, 1

Mo,H.J., McGaugh,S., Bothun,G.D., 1994 MNRAS 267, 129

Nilson,P., 1973, Uppsala General Catalogue of Galaxies, Nova Acta Reginae Soc. Sci. Uppsalinisis Ser. V: A Vol. 1 (UGC).

Popescu,C.C., Hopp,U., Hagen,H.-J., Elsaesser,H., (paper 2, 1996 A $\& A S$ 116, 1 Popescu,C.C., Hopp,U., Elsaesser,H., (paper 4, 1997 A $\mathscr{E} A$ submitted

Pustil'nik,S.A., Ugryumov,A.V., Lipovetsky, A.A., Thuan,T.X., Guseva,N.G., 1995 ApJ 443, 499

Roennback,J., Bergvall,N., 1996, A $\& A$ 302, 353

Rosenberg,J.L. et al. $1994 A J \mathbf{1 0 8}, 1557$

Salzer,J.J. 1989 ApJ 347, 152

Saunders,W., Frenk,C., Rowan-Robinsom,M., Efstathiou,G., Lawrence,A., Ellis,R., Crawford,J., Xia,X.Y., Parry,I., 1991, Nature 349, 32

Schmidt, K.H., Boller, T., 1992, AN 313, 329

Schombert,J.M., Pildes,R.A., Eder,J.A., 1997, ApJS accepted

Slezak,E., de Lapparent,V., Bijaoui,A., 1993, ApJ 409, 517

Soifer,B.T., Houck,J.R., Neugebauer,G., 1987, ARA $\xi A$ 25, 187

Szomoru,A., van Gorkom,J.H., Gregg,M.D., Strauss,M.A., 1996, AJ 111, 2150

Thuan,T.X., Gott,J.R., Schneider,S.E., 1987, ApJ 315, L93

Vennik,J., Hopp,U., Kovachev,B., Kuhn,B., Elsaesser,H., paper 5, 1996 A\&AS $\mathbf{1 1 7}, 261$

Vogeley,M.S., Geller,M.J., Park,C., Huchra,J.P., 1994, AJ 108, 745

Voges,W., Aschenbach,B., Boller,Th., Bruninger,H., Briel,U., Burkert,W., Dennerl,W., Englhauser,J., Gruber,R., Haberl,F., Hartner,G., Hasinger,G., Kürster,M., Pfeffermann,E., Pietsch,W., Predehl,P., Rosso,C., Schmitt,J.H.M.M., Trümper,J., Zimmermann,H.-U., 1996, $A \mathscr{E} A$, in press.

Weistrop,D., Hintzen,P., Liu,C., Lowenthal,J., Cheng,K.-P., Oliversen,R., Brown,L., Woodgate, B., 1995, AJ 109, 981

Ugrymov,A., Engels,D., Lipovetsky,V., Hopp,U., Richter,G., Izotov,Y.I., Kniazev,A.Y., Popescu,C.C., 1997, Proc. Symp. IAU 179, in press

Zwicky, F., Herzog,E., Wild,P., 1961-1968, Catalogue of Galaxies and Clusters of Galaxies, Speich, Zürich (CGCG). 
VOID GALAXIES 